AmJ Physiol Heart Circ Physiol 278: H1571-H1576, 2000.

\title{
Bradykinin mediates cardiac preconditioning at a distance
}

\author{
REGIEN G. SCHOEMAKER AND CAROLINE L. VAN HEIJ NINGEN \\ Department of Pharmacology, Faculty of Medicine and Health Sciences, \\ Erasmus University, NL-3000 DR Rotterdam, The Netherlands
}

\begin{abstract}
Schoemaker, Regien G., and Caroline L. van Heijningen. Bradykinin mediates cardiac preconditioning at a distance. Am J Physiol Heart Circ Physiol 278: H1571-H1576, 2000.-Preconditioning the heart by brief coronary (CAO) or mesenteric artery occlusion (MAO) can protect against damage during subsequent prolonged CAO and reperfusion. The role of bradykinin (BK) in remote cardiac preconditioning by $M A O$ is investigated by antagonizing the $B K B_{2}$ receptor [Hoechst 140 (HOE-140)] or simulating local BK release by mesenteric intra-arterial infusion. Anesthetized male Wistar rats ( $n=6-8$ ) were treated with HOE-140 or saline before starting the preconditioning protocol, CAO, MAO, or non-preconditioned control. I nfarct size related to risk area [ratio of infarct area to area at risk (I A/AR)] was determined after $3 \mathrm{~h}$ of reperfusion following a 60 -min CAO. IA/AR was $62 \pm 5 \%$ in controls and not affected by HOE-140 (58 $\pm 6 \%$ ). CAO as well as MAO significantly protected the heart (IA/AR, $37 \pm 3$ and $35 \pm 5 \%$ ), which was prevented by HOE-140 (IA/AR, $71 \pm 6$ and $65 \pm 7 \%$, respectively). Brief intramesenteric BK infusion mimicked MAO (IA/AR, $26 \pm 3 \%)$. Pretreatment with hexamethonium could abolish this protection (IA/AR, $67 \pm$ 4\%). These data indicate an important role for BK in remote preconditioning by MAO. Results support the hypothesis that remote preconditioning acts through sensory nerve stimulation in the ischemic organ.
\end{abstract}

sensory nerves; mesenteric vascular bed

ISCHEMIC PRECONDITIONING has been described for the myocardium (13) as well as for many other organs, including brain (8), kidney (23), liver (10), and skeletal muscle (12), indicating a rather general phenomenon. However, within the heart, brief local ischemia not only preconditioned that same area but al so adjacent myocardium was protected (15). Moreover, we showed that even brief ischemia in organs other than the heart was able to protect the heart (3) (also termed as remote preconditioning). This has been confirmed by others $(14,20)$. The mechanism within the heart showed similarities with the classical preconditioning $(14,20)$. Outside the heart we showed that for the phenomenon of remote cardiac preconditioning reperfusion of the stimulated organ is required before coronary artery occlusion (CAO) and that protection can beabolished by ganglion blockade with hexamethonium, thus indicat-

The costs of publication of this article were defrayed in part by the payment of page charges. The article must therefore be hereby marked "advertisement" in accordance with 18 U.S.C. Section 1734 solely to indicate this fact. ing a neurogenic pathway that is activated during reperfusion (3). We hypothesized that reperfusion of the ischemic organ locally stimulates sensory nerves, which project to efferent nerves on the myocardium. One of the endogenous substances that is released during ischemia-reperfusion and capable of activating sensory nerves is bradykinin (BK). We herein investigate the role of $\mathrm{BK}$ in remote cardiac preconditioning by mesenteric artery occlusion (MAO). To inhibit the effects of endogenous $B K$, the $B K B_{2}$ receptor, responsible for afferent nerve stimulation, is blocked by its antagonist Hoechst 140 (HOE-140). To mimic the effects of local BK release, the effects of intramesenterically infused BK (7) to locally stimulate afferent nerves in the absence or presence of ganglion blockade were investigated.

\section{MATERIALS AND METHODS}

At all stages the experiments conform with the Guide for the Care and Use of Laboratory Animals. Male Wistar rats (Harlan; Zeist, The Netherlands) weighing 280-380 g at time of the experiment were used. Animals were housed in groups of two or three, placed on a 12:12-h light-dark cycle, and given food and water ad libitum.

Surgical and experimental preparation. Rats were anesthetized with pentobarbital sodium (60 mg/kg ip) and intubated (PE-240) for positive pressure ventilation (Harvard Rodent Ventilator; Hilliston, MA) with room air (60- 70 strokes/min, tidal volume $3 \mathrm{ml}$ ). The abdominal aorta was cannulated via the femoral artery to register mean arterial blood pressure (MAP) and heart rate (HR). A catheter was placed in the femoral vein for infusion of pentobarbital sodium to maintain anesthesia $\left(0.15 \mathrm{mg} \cdot \mathrm{kg}^{-1} \cdot \mathrm{min}^{-1}\right)$ and injection of $\mathrm{HOE}-140$ or saline. An additional catheter was positioned in the right atrium via the right jugular vein for infusion of Haemaccell (Behringwerke; Marburg, Germany) to compensate for cardiac effects of (surgery associated) fluid loss by maintaining right ventricular filling pressure at $5 \mathrm{mmHg}$. Then, the fourth inter costal space was opened, and a 6-0 silk suture was looped under the left descending coronary artery for later induction of CAO $(3,16)$. The abdominal cavity was opened, the anterior mesenteric artery was freed from surrounding tissue, and a loose suture was placed around the artery to facilitate later MAO with an atraumatic clamp (see Ref. 3 for details). Core temperature was monitored continuously using an electronic thermometer (Electromedics) and was maintained between 36.5 and $37.5^{\circ} \mathrm{C}$ by either heating pads or ice-filled packages.

Experimental protocol BK blockade. After surgical preparation, rats were allowed 30 min for stabilization before baseline hemodynamics were recorded. Rats were subdivided to undergo one of six protocols (Fig. 1). All protocols included a 60-min CAO, followed by 180 min of reperfusion. This was preceded by either no preconditioning (protocols 1 and 2), 
Stimulus

Fig. 1. Schematic presentation of 6 protocols in which effect of Hoechst $140(\mathrm{HOE}-140 ; \boldsymbol{\nabla})$ on risk area and infarct size was studied. $\mathbf{\Lambda}$, Measurements of heart rate and blood pressure. CAO, coronary artery occlusion; MAO, mesenteric artery occlusion; Rep, reperfusion.
1. control

3. 15 min $\mathrm{CAO}+10$ min Rep

5. 15 min $\mathrm{MAO}+10 \mathrm{~min}$ Rep

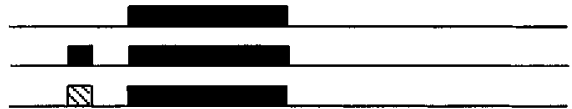

Stimulus

2. control

4. 15 min $\mathrm{CAO}+10$ min Rep

6. 15 min $\mathrm{MAO}+10 \mathrm{~min}$ Rep

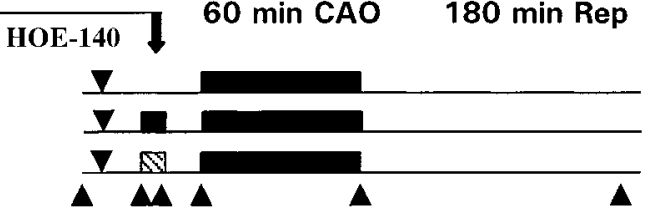

classic preconditioning by a 15-min CAO followed by a 10-min reperfusion (protocols 3 and 4), or by remote preconditioning by a 15-min MAO followed by a 10-min reperfusion (protocols 5 and 6). The experiments were performed in the absence (protocols 1, 3, and 5) or presence (protocols 2, 4, and 6) of $B_{2}$ receptor blockade by HOE-140 (300 $\mu \mathrm{g} / \mathrm{kg})$ administered intravenously $10 \mathrm{~min}$ before preconditioning. The 15-min CAO that is used as preconditioning stimulus, by itself induces only negligible necrosis of $3 \pm 1 \%$ (3). HR and MAP were recorded continuously and analyzed at the time points depicted in Fig. 1. Rats that fibrillated during ischemia or reperfusion were allowed to complete the protocol when conversion to normal sinus rhythm occurred spontaneously or when manual resuscitation was successful within 2 min after onset of fibrillation. Occlusion and reperfusion were visually verified by appearance and disappearance of myocardial or small intestinal cyanosis.

Experimental protocol BK stimulation. Surgical preparation was slightly adjusted to include intramesenteric infusion of BK, in that no suture was placed around the mesenteric artery but one of the side branches was cannulated with the catheter tip positioned at the edge of the mainstream of the mesenteric artery (7). After surgical preparation, rats were allowed $30 \mathrm{~min}$ for stabilization before baseline hemodynamics were recorded. Rats were subdivided for three protocols (Fig. 2). All protocols again included $60 \mathrm{~min}$ of $\mathrm{CAO}$, followed by $180 \mathrm{~min}$ of reperfusion. This was preceded by $5 \mathrm{~min}$ of intramesenteric infusion of saline or BK, followed by 5 min of rest. BK was infused at $1 \mu \mathrm{g} / \mathrm{min}$, which is reported to stimulate sensory neurons without direct systemic hemodynamic effects (7). Whether the effects of intramesenteric BK infusion on myocardial infarct size could indeed be attributed to nerve stimulation rather than to circulating BK was further verified by blockade of the nervous pathway by hexamethonium ( $20 \mathrm{mg} / \mathrm{kg}$ iv) $15 \mathrm{~min}$ before the intramesenteric BK infusion. HR and MAP were recorded continuously and analyzed at the time points shown in Fig. 2.

Measurement of area at risk and infarcted area. At the end of each experiment the heart was quickly removed and placed in ice-cold saline before it was mounted on a modified Langendorff apparatus and perfused with cold saline via the aorta to wash out the blood. After the coronary artery ligature was retied, the heart was perfused with trypan blue $(0.4 \%$,
Sigma Chemical) to stain the perfused myocardium blue, whereas the area at risk (AR) remains unstained. The heart was placed at $-80^{\circ} \mathrm{C}$ for $10 \mathrm{~min}$ before it was cut in slices (1 $\mathrm{mm}$ thickness) from apex to base. From each slice, the right ventricle was removed and the left ventricle (LV) including the septum was divided into the $A R$ and the remaining LV. The AR was then incubated for $10 \mathrm{~min}$ in $37^{\circ} \mathrm{C}$ nitro blue tetrazolium (Sigma Chemical; 1 mg/ml Sörensen buffer, $\mathrm{pH}$ 7.4), which stains vital tissue purple but leaves irreversibly damaged tissue unstained [the infarct area (I A)]. After the IA was separated from the noninfarcted area, the right ventricle as well as the different areas of the LV were dried and weighed separately.

Evaluation of dose of HOE-140. To effectively inhibit the effects of BK during the experimental protocol, the dose and duration of HOE-140 were evaluated in separate rats. For that, rats were anesthetized with pentobarbital sodium and catheters were implanted in the femoral artery as well as the femoral vein (for detailed description see Surgical and experimental preparation). The former was connected to a pressure transducer for registration of MAP, whereas the latter was used for intravenous administration. After at least $30 \mathrm{~min}$ of stabilization, rats were injected with BK (4 $\mu \mathrm{g} / \mathrm{kg}$ iv) and blood pressure decrease was measured. This procedure was repeated $5 \mathrm{~min}$ later to examine possible tachyphylaxis. HOE-140 (300 $\mu \mathrm{g} / \mathrm{kg})$ was then injected and the blood pressure response to BK was followed for $4 \mathrm{~h}$.

Data analysis and presentation. Data are presented as means \pm SE unless indicated otherwise. Correlation between $A R$ and IA was evaluated by linear regression analysis. Differences in the IA-to-AR ratios (IA/RA) between groups were analyzed using one-way ANOVA followed by Bonferroni's t-tests for multiple group comparisons. HR and MAP for the different groups were analyzed by two-way ANOVA for repeated measurements. Differences were regarded statistically significant at $\mathrm{P}<0.05$.

\section{RESULTS}

Mortality. A total of 71 rats entered the study. Fifty-two of these rats completed their protocol and have been included in the final analysis. The main cause of exclusion was premature death (17 rats) due to
Fig. 2. Schematic presentation of 3 protocols in which effect of intramesenteric bradykinin (BK) in absence or presence of hexamethonium ( $\mathrm{Hex}, \boldsymbol{\nabla})$ on risk area and infarct size was studied. $\boldsymbol{\Lambda}$, Measurements of heart rate and blood pressure.

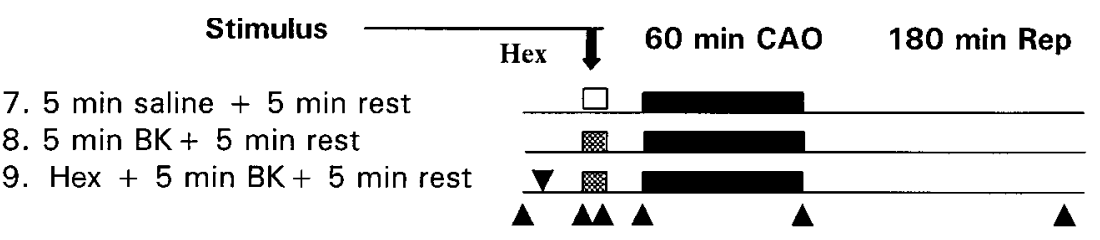




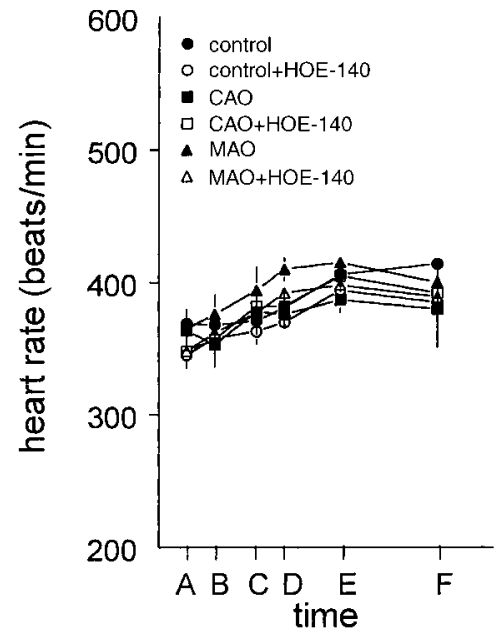

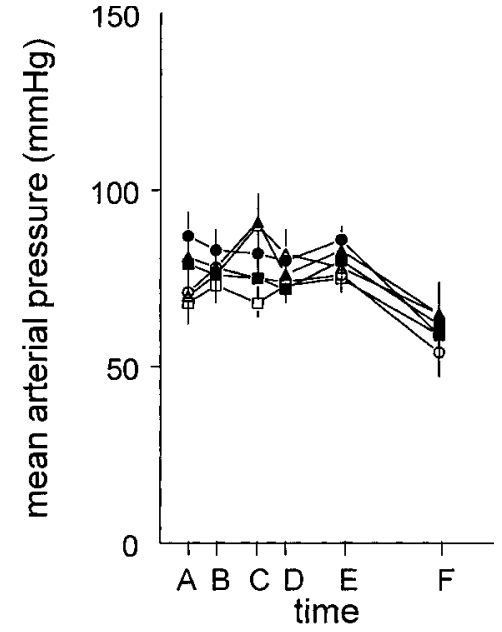

Fig. 3. Heart rate and blood pressure at different time points in protocols described in Fig. 1: A, after $30 \mathrm{~min}$ of stabilization; B, 10 min after injection of saline or HOE-140; C, at end of 15-min control, coronary or mesenteric artery occlusion; $D$, after 10 min of reperfusion; $\mathrm{E}$, at end of 60-min CAO; F, at end of 3-h coronary reperfusion. sustained ventricle fibrillation and/or hypotension during the 180-min reperfusion period.

Evaluation of dose of HOE-140. BK, administered intravenously at a dose of $4 \mu \mathrm{g} / \mathrm{kg}$, decreased MAP by $23 \pm 3 \mathrm{mmHg}$. The second administration 5 min later induced similar MAP reduction, indicating notachyphylaxis. HOE-140 by itself had no effect on MAP or HR but completely blocked the response to BK for at least 90 min. At 120 min the response was still inhibited by $70 \%$, whereas at 210 min the response to BK was fully restored.

BK blockade. Hemodynamics were registered continuously by means of MAP and HR. Significant differences were not observed for MAP or for $\mathrm{HR}$ among the protocols at any time point (F ig. 3). Moreover, administration of HOE-140 or ischemia and reperfusion did not cause significant changes in HR and MAP.

Without preconditioning, a significant correlation between AR and IA is observed (regression line, IA =
0.54; RA, $+6.25 ; r=0.894)$. Pretreatment with HOE140 does not change that (Fig. 4), resulting in similar IA/AR as presented in Table 1.

Remote preconditioning by MAO resulted in pronounced cardiac protection, as indicated by the position of the points with respect to the regression line of control hearts in Fig. 4. This cardiac protection was completely abolished by HOE-140 (Fig 4). Accordingly, IA/AR was significantly lower after remote preconditioning and normalized by HOE-140 (Table 1).

Classic preconditioning by CAO resulted in similar protection as remote preconditioning and was also completely abolished by HOE-140. This was substantiated by the IA/AR (see Table 1).

BK stimulation. Saline infusion in the mesenteric vascular bed did not affect MAP, HR, or infarct ratio, and was similar to the control group of protocol 1 . Therefore, data of intramesenteric BK infusion were compared with the control data of protocol 1. Although
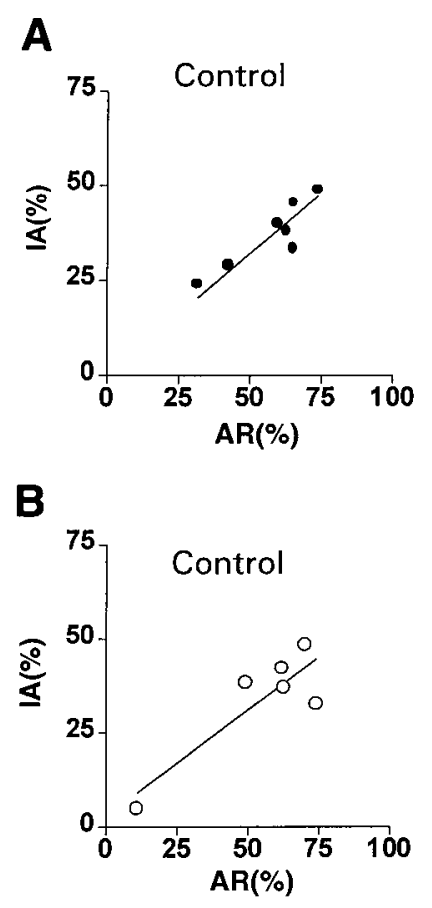
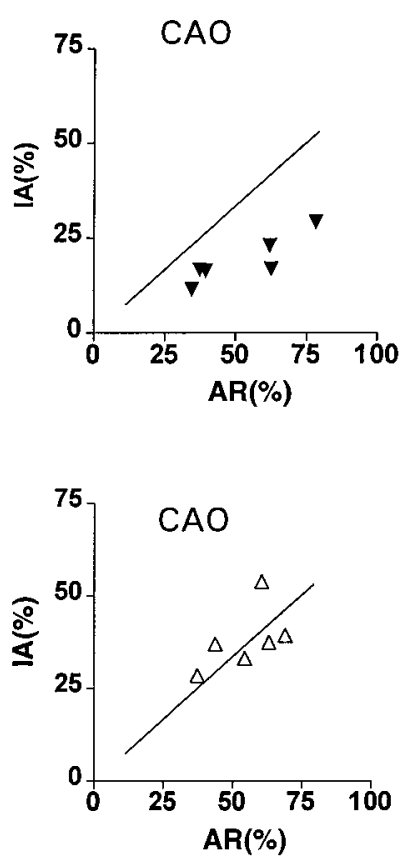
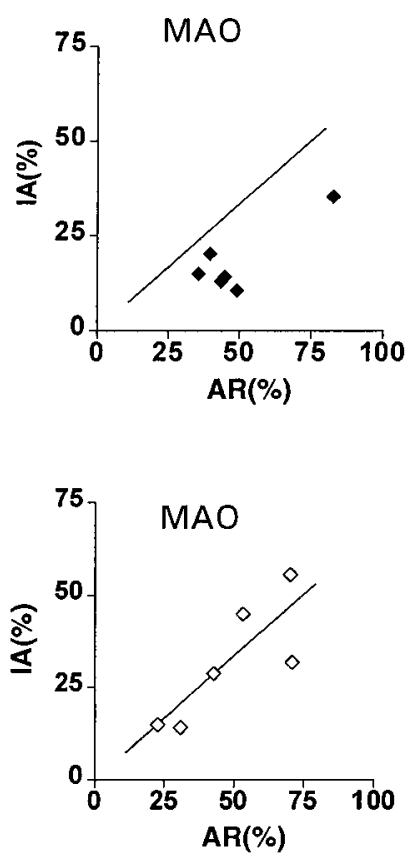

Fig. 4. Infarct area (IA) plotted against area at risk (AR) as percentage of left ventricular weight for different protocols. Regression line for controls is de picted in left panel of A. Because HOE140 did not change regression line for controls in remaining panels, combined regression line for controls is displayed. A: without $\mathrm{HOE}-140$ treatment. B: with HOE-140 treatment. 
Table 1. IA-to-AR ratio for different protocols

\begin{tabular}{llll}
\hline \hline & $\mathrm{n}$ & AR, \%LV & IA/AR \\
\hline Control & 8 & $60 \pm 5$ & $62 \pm 5$ \\
Control + HOE-140 & 6 & $50 \pm 9$ & $58 \pm 6$ \\
CAO & 6 & $52 \pm 7$ & $37 \pm 3^{*}$ \\
CAO+ HOE-140 & 6 & $55 \pm 5$ & $71 \pm 6 \dagger$ \\
MAO & 6 & $52 \pm 7$ & $35 \pm 5^{*}$ \\
MAO + HOE-140 & 6 & $49 \pm 8$ & $65 \pm 7 \dagger$ \\
Saline & 3 & $53 \pm 14$ & $52 \pm 4$ \\
Bradykinin & 7 & $55 \pm 5$ & $26 \pm 3^{*}$ \\
Bradykinin + Hex & 6 & $50 \pm 5$ & $67 \pm 4 \dagger$ \\
\hline
\end{tabular}

Values are means $\pm \mathrm{SE} ; \mathrm{n}=$ no. of rats studied. $\mathrm{AR}$, area at risk; IA, infarct area; CAO, classic preconditioning by coronary artery occlusion; MAO, remote preconditioning by mesenteric artery occlusion; HOE-140, H oechst 140 pretreatment; Hex, hexamethonium pretreatment. *Significant effect compared with control. †Significant effect of HOE-140 or Hex pretreatment on respective cardiac protection.

transiently BK infusion caused a slight increase in HR and MAP, steady-state hemodynamics after $5 \mathrm{~min}$ of infusion were not significantly altered. Pretreatment with hexamethonium caused a significant reduction in $\mathrm{HR}$ and MAP, which was not further altered by BK infusion (Fig. 5).

Whereas intramesenteric infusion of saline had no effect on infarction of the heart, $5 \mathrm{~min}$ of intramesenteric BK infusion, followed by 5 min of rest before the 60-min CAO resulted in significant cardiac protection (Table 1). Protection was abolished by pretreatment with the ganglion blocker hexamethonium.

\section{DISCUSSION}

In the present study weinvestigated the role of BK in the remote cardiac preconditioning by mesenteric artery occlusion-reperfusion. The working hypothesis was that BK that is locally released during ischemiareperfusion in the mesenteric bed stimulates sensory nerves projecting on efferent nerves to the heart. The hypothesis is supported by our findings that 1 ) HOE140 , an antagonist for the $B_{2}$ receptor involved in sensory nerve stimulation, completely abolished the cardiac protection by mesenteric ischemia-reperfusion; 2) intramesenteric BK, at a dose that stimulates sen- sory nerves without systemic effects, could mimic cardiac protection by mesenteric ischemia-reperfusion; and 3 ) the protective effects of intramesenteric BK can be completely blocked by the ganglion blocker hexamethonium.

BK blockade. The $B_{2}$ receptor is reported to be the receptor involved in vasodilation as well as sensory nerve stimulation (9) and can be antagonized by HOE140. In pentobarbital sodium-anesthetized rats, intravenous BK induces a maximal decrease in MAP of $\sim 25$ $\mathrm{mmHg}$ at a dose of $10 \mu \mathrm{g} / \mathrm{kg}$ (pilot studies). In the present study $4 \mu \mathrm{g} / \mathrm{kg}$ were used as a submaximal dose to examine the time course of $\mathrm{BK}$ response inhibition after $300 \mu \mathrm{g} / \mathrm{kg} \mathrm{HOE}-140$ (19). HOE-140 was capable of blocking the response to BK completely for at least 90 min, which meant that the preconditioning protocols were performed under $\mathrm{B}_{2}$ blockade up until at least the 60-min CAO. The effect of HOE-140 is waning during the 180-min reperfusion period.

Pretreatment with $\mathrm{HOE}-140$ had no effect on infarct size of non-preconditioned rats. This is in general accordance with earlier findings in other studies $(2,4)$. However, HOE-140 completely abolished the cardiac protection of the remote (by MAO) as well as the classic (by $\mathrm{CAO}$ ) preconditioning. Attenuation of classic preconditioning by HOE-140 has been reported before but appeared to occur in vivo $(4,21)$ rather than in vitro $(2$, 18). Moreover, cardiac protection could be obtained by intracoronary BK infusion in vitro $(2,18)$ as well as in vivo (21). In our previous study (3), in contrast to remote preconditioning, classic preconditioning could not beinhibited by hexamethonium nor had hexamethonium itself cardioprotective effects. Thus although sensory nerves may be present on the ventricle, which could induce efferent nerve stimulation to the heart, results suggest a local effect of $\mathrm{BK}$ on the heart rather than the aforementioned neurogenic pathway. This would be in accordance with the study of Goto et al. (4), who suggested that blood-bornekininogens rather than autonomic nerves would beinvolved in classic preconditioning.
Fig. 5. Heart rate and blood pressure at different time points in protocols described in Fig. 2: A, after 30 min of stabilization; $B, 10 \mathrm{~min}$ after injection of saline or hexamethonium; $\mathrm{C}$, at end of 5-min intramesenteric saline or BK infusion; $D$, just before 60-min CAO; $E$, at end of 60-min CAO; F, at end of 3-h coronary reperfusion.
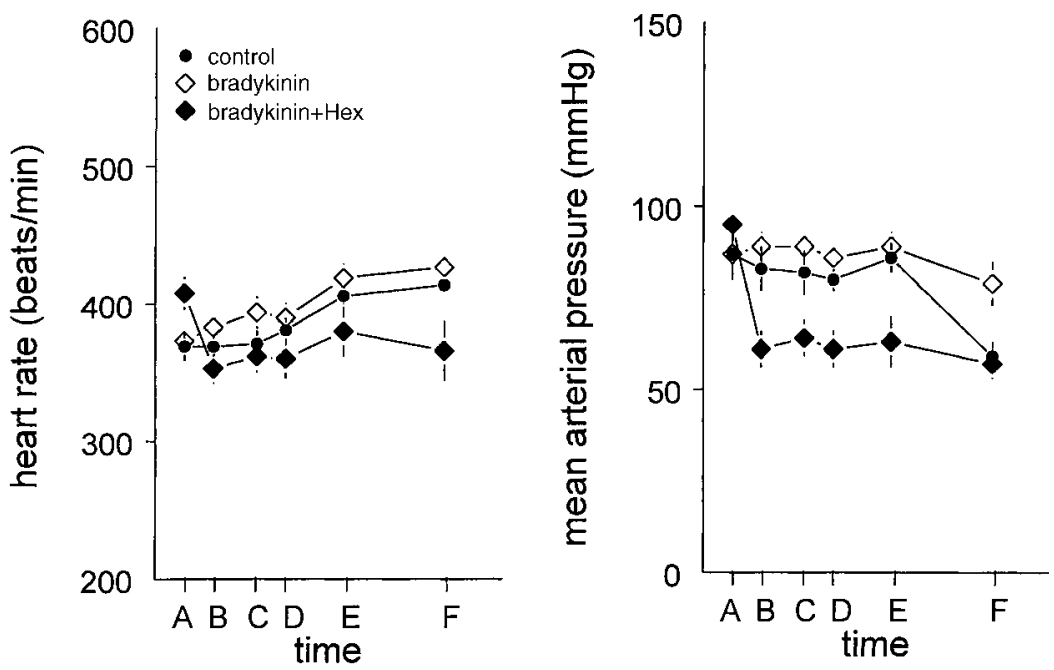
In contrast, remote preconditioning could well be associated with stimulation of a neurogenic pathway, because cardiac protection can be attenuated through ganglion blockade by hexamethonium (3). However, similar to classic preconditioning, pretreatment with HOE-140 completely abolished the cardiac protection of $15 \mathrm{~min}$ of MAO. This result indicates that endogenous BK has an important role in the neurogenic pathway of remote preconditioning.

Locally infused BK in the mesenteric vascular bed could mimic remote cardiac preconditioning by reduction of myocardial infarct size. The calculated local concentration of BK $(1 \mu \mathrm{g} / \mathrm{min}$ BK into $\pm 15 \mathrm{ml} / \mathrm{min}$ mesenteric flow results in $67 \mu \mathrm{g} / \mathrm{l}=55 \mathrm{nM}$ ) of $55 \mathrm{nM}$ at intramesenteric infusion closely resembles the cardiac BK concentrations with classic preconditioning in pigs, from $30 \mathrm{nM}$ at baseline to $60 \mathrm{nM}$ during ischemiareperfusion (17).

The protection could be prevented by ganglion blockade. Because we showed that intravenous injection of BK decreased blood pressure (as we used to determine duration of action of HOE-140) the slight increases of $\mathrm{HR}$ and MAP after intramesenteric BK infusion suggest a locally induced rather than a systemic effect. The cardiac protection observed after intramesenteric BK therefore could be attributed to an action of BK in the mesenteric bed rather than the well-known effects of circulating BK on the heart itself $(2,4,18,21)$.

The route of administration and the dose of local BK in the present study have been reported to stimulate afferent mesenteric nerves (7), but we cannot exclude effects of BK leaking into the systemic circulation. However, the observation that the cardioprotective effect of intramesenteric BK infusion could be prevented by ganglion blockade with hexamethonium suggests a neuronal rather than a circulating mechanism. This is supported by the observation that intramesenteric injection of capsaicin, at a dosethat stimulates but does not desensitize sensory nerves (22), caused similar cardioprotection as BK (data not shown).

However, we cannot exclude one hypothetical mechanism for the remote preconditioning. Because BK also has been reported to have a role in sensory function in other organs and even up to the level of the spinal cord (11), which may include hexamethonium-sensitive pathways as well, intramesenteric infused BK could have leaked out to other organs, including the heart or the spinal cord, and have exerted its neuronal stimulation there.

In addition to a direct role of BK in sensory function (11), intramesenteric BK infusion caused a substantial reduction of mesenteric blood flow (7). Regarding the slightly increased MAP, this may result in $\pm 40 \%$ increase in mesenteric vascular resistance at the dose of BK used (7). This by itself could cause mesenteric ischemia, which, as we have shown, may result in cardiac protection (present study and Ref. 3). Thus whether intramesenteric endogenous or exogenous BK directly stimulates afferent nerves or acts in association with mesenteric ischemia-reperfusion is not known.
The mechanism at the other end of the neurogenic pathway, that is on the heart, is not yet clear. I wamoto and co-workers (6) indicate that the heart can be preconditioned by stimulation of the left stellate cardiac nerve, if simultaneously the increase in coronary blood flow was restricted. Moreover, cardiac preconditioning can be achieved by increasing endogenous catecholamine release (1). These studies indicate that under certain conditions the sympathetic nervous system may have a role in cardiac preconditioning. Moreover, two recent studies $(14,20)$ indicate that cardiac adenosine receptors may be involved in remote preconditioning by renal artery occlusion-reperfusion. Adenosine activates a pertussis toxin-sensitive inhibitory $\mathrm{G}$ protein, which can facilitate the activation of ATPsensitive $\mathrm{K}^{+}$channels. Stimulation of $\alpha$-adrenoceptors by endogenous catecholamines can exert preconditioning effects through the activation of protein kinase $C$ via such a G protein (5). However, further investigation is needed for its role in remote preconditioning.

In conclusion, data from the present study showed evidence for an important rol e for BK in remote preconditioning. Results support the hypothesis that remote preconditioning could act through sensory nerve stimulation in the ischemic organ. The consecutive mechanism within the heart is not yet clear but may involve the endogenous rel ease of catecholamines.

HOE-140 was a generous gift of Hoechst, Germany.

Address for reprint requests and other correspondence: R. G. Schoemaker, Dept. of Pharmacology, Erasmus Univ. Rotterdam, Dr. Molewaterplein 50, 3015 GE Rotterdam, The Netherlands (E-mail: schoemaker@farma.fgg.eur.nl).

Received 14J uly 1999; accepted in final form 5 November 1999.

\section{REFERENCES}

1. Bankwala Z, Hale SL, and Kloner RA. Alpha-adrenoceptor stimulation with exogenous norepinephrine or release of endogenous catecholamines mimics ischemic preconditioning. Circulation 90: 1023-1028, 1994.

2. Bugge $\mathbf{E}$ and Ytrehus $\mathbf{K}$. Bradykinin protects against infarction but does not mediate ischemic preconditioning in the isolated rat heart. J Mol Cell Cardiol 28: 2333-2341, 1996.

3. Gho BC, Schoemaker RG, van den Doel MA, Duncker DJ , and Verdouw PD. Myocardial protection by brief ischemia in noncardiac tissue. Circulation 94: 2193-2200, 1996.

4. Goto M, Li iu Y, Yang XM, Ardell J L, Cohen MV, and Downey J M. Role of bradykinin in protection of ischemic preconditioning in rabbit hearts. Circ Res 77: 611-621, 1995.

5. Hu K and Nattel S. Mechanisms of ischemic preconditioning in rat hearts. I nvolvement of alpha 1B-adrenoceptors, pertussis toxin-sensitive $\mathrm{G}$ proteins, and protein kinase C. Circulation 92: 2259-2265, 1995

6. I wamoto T, Bai XJ , and Downey HF. Preconditioning with supply-demand imbalancelimits infarct size in dog heart. Cardiovasc Res 27: 2071-2076, 1993.

7. J anssen BJ , van E ssen H, Struyker Boudier HA, and Smits J F. Hemodynamic effects of activation of renal and mesenteric sensory nerves in rats. Am J Physiol Regulatory Integrative Comp Physiol 257: R29-R36, 1989.

8. Kitagawa $K$, Matsumoto $M$, Tagaya $M$, Hata $R$, Ueda $\mathbf{H}$, Niinobe M, Handa N, Fukunaga R, Kimura K, and Mikoshiba K. "Ischemic tolerance" phenomenon found in the brain. Brain Res 528: 21-24, 1990.

9. Lew WY and Longhurst J C. Substance P, 5-hydroxytryptamine, and bradykinin stimulate abdominal visceral afferents. Am J Physiol Regulatory Integrative Comp Physiol 250: R465R473, 1986. 
10. Lloris-Carsi J M, Cejalvo D, Toledo-Pereyra LH, Calvo MA, and Suzuki S. Preconditioning: effect upon lesion modulation in warm liver ischemia. Transplant Proc 25: 3303- 3304, 1993.

11. Lopes $\mathbf{P}$ and Couture R. Localization of bradykinin-like immunoreactivity in the rat spinal cord: effects of capsaicin, melittin dorsal rhizotomy and peripheral axotomy. Neuroscience 78: 481497, 1997.

12. Mounsey RA, Pang CY, and Forrest C. Preconditioning: a new technique for improved muscle flap survival. Otolaryngol Head Neck Surg 107: 549-552, 1992.

13. Murry CE, J ennings RB, and Reimer KA. Preconditioning with ischemia: a delay of lethal cell injury in ischemic myocardium. Circulation 74: 1124-1136, 1986.

14. Pell TJ, Baxter GF, Yellon DM, and Drew GM. Renal ischemia preconditions myocardium: role of adenosine receptors and ATP-sensitive potassium channels. Am J Physiol Heart Circ Physiol 275: H1542-H1547, 1998.

15. Przyklenk K, Bauer B, Ovize M, Kloner RA, and Whittaker P. Regional ischemic "preconditioning" protects remote virgin myocardium from subsequent sustained coronary occlusion. Circulation 87: 893-899, 1993.

16. Schoemaker RG, Debets J J, Struyker-Boudier HA, and Smits J F. Delayed but not immediate captopril therapy improves cardiac function in conscious rats, following myocardial infarction. J Mol Cell Cardiol 23: 187-197, 1991.
17. Schulz R, Post H, Vahlhaus C, and Heusch G. Ischemic preconditioning in pigs: a graded phenomenon; its relation to adenosine and bradykinin. Circulation 98: 1022-1029, 1998.

18. Starkopf J , Bugge E, and Ytrehus K. Preischemic bradykinin and ischaemic preconditioning in functional recovery of the gl obally ischaemic rat heart. Cardiovasc Res 33: 63- 70, 1997.

19. Sun $\mathbf{W}$ and Wainwright $\mathbf{C L}$. The potential antiarrhythmic effects of exogenous and endogenous bradykinin in the ischaemic rat heart in vivo. Coron Artery Dis 5: 541-550, 1994.

20. Takaoka A, Nakae I, Mitsunami K, Yabe T, Morikawa S, Inubushi T, and Kinoshita M. Renal ischemia/reperfusion remotely improves myocardial energy metabol ism during myocardial ischemia via adenosine receptors in rabbits: effects of "remote preconditioning". J Am Coll Cardiol 33: 556-564, 1999.

21. Wall TM, Sheehy R, and Hartman J C. Role of bradykinin in myocardial preconditioning. J Pharmacol Exp Ther 270: 681689, 1994.

22. Warzecha Z, Dembinski A, J aworek J, Ceranowicz $\mathbf{P}$, Szlachcic A, Walocha J , and Konturek SJ . Role of sensory nerves in pancreatic secretion and caerulein-induced pancreatitis. J Physiol Pharmacol 48: 43-58, 1997.

23. Zager RA, Baltes LA, Sharma HM, and J urkowitz MS. Responses of the ischemic acute renal failure kidney to additional ischemic events. Kidney Int 26: 689- 700, 1984.

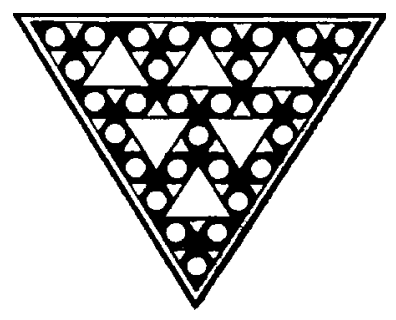

